\title{
Efficacy and safety of Dynamic Quadripolar Radio-Frequency, a new high-tech, high-safety option for vulvar rejuvenation
}

\author{
Gianluca Benincà ${ }^{1}$, David Bosoni ${ }^{2}$, Franco Vicariotto ${ }^{3}$ and Mauro Raichi ${ }^{4 *}$ \\ ${ }^{1}$ General Surgeon, Plastic and Aesthetic Surgeon, Full Professor L.U.de.S, University of Lugano, Lugano, Switzerland, \\ ${ }^{2}$ Research Centre for Reproductive Medicine, Gynecological Endocrinology and Menopause, Section of Obstetrics and Gynecology, Department of Clinical, \\ Surgical, Diagnostic and Pediatric Sciences, University of Pavia, Pavia, Italy \\ ${ }^{3}$ Italian Menopause Society (SIM) \\ ${ }^{4}$ Clinical Pharmacology and Biophysics Consultant, Milan, Italy
}

\begin{abstract}
Background: The flow of papers about surgical and non-surgical vulvar rejuvenation techniques parallels the steadily increasing interest by the general public and the market. All vulvar rejuvenation procedures share the goal of correcting vulvar aesthetic imperfections and alleviating the related physical and psychological burden experienced by the woman in her everyday life (irritations, discomfort, possibly unrewarding couple relationship). Dynamic Quadripolar RadioFrequency (DQRF) is the latest-born technology in the evolving world of light- and energy-based therapies as effective alternative options to traditional techniques of aesthetic and cosmetic surgery.

Methods: More than 500 complete DQRF vulvar rejuvenation cycles were performed between March 2016 and June 2017 according to the proprietary "EVA Vulvar Rejuvenation" treatment protocol in an advanced international centre of plastic and aesthetic medicine and surgery. The evolution of vulvar aesthetics and the subjective level of gratification of women for aesthetic and daily life benefits were retrospectively evaluated in a random sample of 25 DQRF cycles by the same $\mathrm{EVA}^{\mathrm{TM}}$ operator. As regards efficacy, for each woman the authors retrospectively scored, on 10-cm visual analogue scales (VAS), the photographic documentation of the vulvar area before and at the end of the DQRF rejuvenation cycle and after 3 months of follow-up without further treatments. While scoring, authors were blind to the history and demographic details of women.

The outcomes (VAS scores) of standardised-format interviews conducted by the EVA ${ }^{\mathrm{TM}}$ operator at the end of each vulvar rejuvenation session were also analysed. Investigated issues: wellbeing during the procedure and aesthetic and functional benefits experienced by the woman up to that moment of the vulvar rejuvenation cycle.

Results: Improvements of vulvar aesthetics were objectively apparent in all women at the end of the DQRF rejuvenation sessions, often after the first one. Mean scores attributed to the photographic documentation of the vulvar area significantly improved between the beginning and the end of treatments (4.1 vs. 7.8 ; $<<0.05)$. Aesthetic objective improvements persisted over the following months (score at the end of the no-treatment follow-up: 7.6). The level of individual gratification of treated women, already significantly increased before the second DQRF session, steadily increased over the following weeks and after the end of their vulvar rejuvenation cycle. No woman experienced clinically significant adverse effects; only a slight degree of transitory hyperaemia was commonly reported.

Conclusions: A short vulvar rejuvenation cycle of four 10-min sessions based on the new DQRF technology significantly improves vulvar aesthetics and helps to suppress the problems and discomfort in the woman's everyday life that are commonly related to her vulvar atrophy. Aesthetic and functional progress is seen in all treated women; relief of discomfort and irritations was often reported even before the end of the DQRF sessions. The procedure is comfortably office-based, technically simple and devoid of disturbing adverse effects. Development of the DQRF technology in the next future will have to focus on cytological and histological studies to deepen understanding of biological effects, as well as on expanding the number of treated women and the documented follow-up period (so far, one year in published clinical studies). Validated questionnaires will have to be used to assess the subjective level of gratification of treated women.
\end{abstract}

\section{Introduction}

According to the American College of Obstetricians and Gynecologists, aesthetic and cosmetic surgery over the vulvar area is experiencing double-digit growth in the United States, even in young and sometimes adolescent women [1]. This is no more than one example of the growing interest that aesthetic (cosmetic) gynaecology is currently enjoying all over the world from both technical and scientific perspectives and the business point of view.

This booming world is borderline with the technologies and procedures aimed at relieving the symptoms and discomfort associated with the genitourinary syndrome of menopause - postmenopausal vaginal dryness, pain, burning and itching, dyspareunia, slight urinary incontinence and recurrent urinary tract infections - and, in younger women, with post-delivery vaginal laxity. Both these conditions impact

Correspondence to: Mauro Raichi, Clinical Pharmacology and Biophysics Consultant, Milan, Italy, E-mail: mraichi@gmail.com

Key words: Aesthetic gynaecology, Radiofrequency, Vulvar rejuvenation

Received: November 02, 2017; Accepted: November 21, 2017; Published: November 27, 2017 
on the woman's self-perception and self-confidence, and severely challenge the holistic quality of life of affected women [2,3]. However, it is mainly aesthetic gynaecology that at present enjoys centre stage in terms of attention by the general public and media. Two more examples coming from the two shores of the Atlantic: according to a 2010 American survey, looking for improved aesthetics was the only reason leading $90 \%$ of patients to undergo elective surgery for vaginal tightening, vaginoplasty and perineal support [4], whilst elective reductive labiaplasty procedures doubled in the United Kingdom in the decade around the turn of the century [5].

Much the same is true in Italy. According to the 2014 data of the Italian Association for Aesthetic Plastic Surgery (Italian acronym, AICPE), more and more Italian women undergo intimate plastic surgery (3,300 in 2014 or $1.3 \%$ of all aesthetic surgery procedures, $+13 \%$ compared with 2013) [6]. Labiaplasty techniques, autologous adipose tissue transplantation (lipofilling) and office injections of hyaluronic acid fillers are some of the options that are at present available to plastic surgery and aesthetic gynaecology practitioners [7-9].

Attention is also dramatically surging for light- and energybased technologies such as monochromatic laser radiation and radiofrequency thanks to their non-invasive nature, simpler logistics, and reduced costs. Emission of electromagnetic energy of variable wavelength aims at anatomical re-modelling and rejuvenation of extraintroital and extra-vaginal tissues through thermal re-activation of fibroblasts [10-12]. Immunohistochemical and electron microscope observations are steadily accumulating that correlate fibroblast reactivation and deposition of new networks of collagen and elastin fibres in the subepithelial layers of the vulva. Increasing tissue levels of profibrotic cytokine TGF- $\beta 1$ and persistent activation of heat shock proteins are also markers of connective tissue matrix re-modelling [12].

\section{"EVATM Vulvar Rejuvenation" treatment protocol}

- Four 10-min sessions, spaced 14-16 days

- Setting of the radiofrequency generator: $1 \mathrm{Mhz}$

- Operating Power: $8-14 \%$ of the maximum device power (55 W)

- Target temperature in vulvar tissues during procedures: $42^{\circ} \mathrm{C}$ (range $40-43{ }^{\circ} \mathrm{C}$ )

The innovative Dynamic Quadripolar RadioFrequency (DQRF) technology is based on advanced research by the Italian company Novavision Group S.p.A. (Misinto, Monza-Brianza, Italy). Together with the low-energy DQRF-based EVA ${ }^{\text {tx }}$ device and the proprietary "EVA ${ }^{\mathrm{nx}}$ Vulvar Rejuvenation" treatment protocol, DQRF is the most recent technology designed to trigger anatomical re-modelling in vulvar tissues.

The core of DQRF innovation is in the peculiar interaction between the subepithelial layers of the vulva and the energy emitted by the radiofrequency generator. DQRF biophysics allows the operator to define the depth and volume of the target vulvar area and drastically reduce administered energy; electronically controlled movement and temperature sensors in the EVA ${ }^{\mathrm{mi}}$ device (RSS ${ }^{\mathrm{mm}}$, Radiofrequency Safety System, technology) allow rigid control of tissue temperature [13]. The ongoing clinical studies programme begins to suggest that the DQRF technology might in fact overcome the unwieldiness and safety problems of conventional light- and energy-based vulvar rejuvenation devices $[13,14]$.
The herein presented study was designed with a double goal: evaluating the objective evolution of vulvar morphology in a random sample of women with vulvar atrophy treated with the DQRF technology and monitoring the treated women's subjective gratification for the perceived aesthetic and functional benefits in their everyday life.

\section{Material and Methods}

More than 500 DQRF vulvar rejuvenation cycles were performed between March 2016 and May 2017 at the international centre for plastic and aesthetic medicine and surgery "Naturade Women's Clinic" (Guangzhou, Guangdong, PRC).

All treatments followed the "EVA" Vulvar Rejuvenation" protocol, developed from preclinical data by the DQRF patents holder and producer of the $\mathrm{EVA}^{\mathrm{m}}$ device and validated in the present study. Rigid standardized procedures allowed collection of comparable data ready for statistical analysis. The first author personally supervised all activities of local operators.

The study was carried out in a retrospective random sample of 25 women who had completed their 4-session DQRF vulvar rejuvenation cycle. The sample was selected with the help of a random numbers generator within all women who had completed their DQRF rejuvenation cycle in the centre and had been treated by the same operator (randomly chosen).

All women showing evidence of vulvar dystrophy, acute or chronic vulvar disorders including dermatitis, condylomata and herpes simplex, or considered at high risk for human Papillomavirus infections were excluded from the sample; some visible laxity of labia minora or referred vulvar and/or vestibular dryness did not prevent sampling. Women poorly sensitive to pain or heat or showing areas of vulvovaginal ischemia as well as unrepaired wounds, mucosal or vulvar irritations or signs of infection in the treatment area were similarly excluded. A short standardised interview by the operator had already identified women (retrospectively excluded from sampling) with symptoms related to, or arising suspicion of, immune depression, uncontrolled diabetes mellitus, urinary tract or sexually transmitted infections, moderate or severe pelvic organ prolapse and bleeding diathesis. Women being treated with anti-coagulant or immunosuppressive drugs or radiant therapy had also been preliminarily screened.

Objective aesthetic efficacy was assessed by independent retrospective scoring by authors of the photographic documentation of the vulvar area of each sampled woman before and at the end of the DQRF rejuvenation cycle and after 3 months of follow-up without further treatments; $10 \mathrm{~cm}$ Visual Analogue Scales (VAS) were used for scoring. Evaluators were blind to the history and demographic details of sampled women as well as to the outcomes of past interviews by the operator (see below). Individual author-attributed VAS scores were then averaged to monitor the mean evolution of vulvar aesthetics at each assessment time and compared with a non-parametric test (Wilcoxon Signed Rank Test).

As regards the assessment of subjective benefits perceived by treated women, the analysis was based on first-hand information prospectively collected by the local operator with short standardised interviews before each DQRF treatment session. The operator's standardised questions had focused on both the woman's subjective perception of any improvement of her vulvar aesthetics and the benefits the woman had the sensation to experience in her daily life due to irritation and discomfort (associated for instance with tight trousers 
and lingerie), loss of self-esteem, difficulties in social interactions, and problems with sexual life and couple relationship. At the end of the interview, the operator had asked the woman for an overall categorical assessment of both her subjective aesthetic gratification and perceived functional benefits ("Not at all satisfied", "Poorly satisfied", "Fairly satisfied", "Highly satisfied"; retrospective analysis of the distribution of subjective women's assessments over time: chi-square test).

In those interviews, women were also questioned about comorbilities (see above) and any side effects experienced after the previous DQRF session. Two-sided 95\% confidence levels were used for all statistical tests with $\mathrm{p}<0.05$ as cut-off for significance. All study materials were peer-reviewed for ethical problems; all women had given informed consent to anonymous collection of their data before the first DQRF session.

\section{Results}

The mean age of sampled women was 34.3 years (range, 25-44); in 8 women there was a slight degree of labia minor laxity, in 11 vulvar and/ or vestibular dryness. The photographic documentation of a selection of vulvar atrophy cases before and at different steps during the DQRF "EVA ${ }^{\text {тx }}$ Vulvar Rejuvenation" programme demonstrates with visual evidence the tightening efficacy of the new DQRF technology over the vulvar area even before the final session. On average, the VAS scores related to the overall aesthetic vulvar appearance significantly improved between the beginning and the end of the vulvar rejuvenation program (Figures 1-8).

Follow-up information for the 3-following no-treatment months was available for 22 of the sampled women (88\%); 3 women were lost to follow-up. No significant objective worsening of vulvar aesthetics occurred during the follow-up period in spite of lack of further rejuvenation sessions (Figure 9).
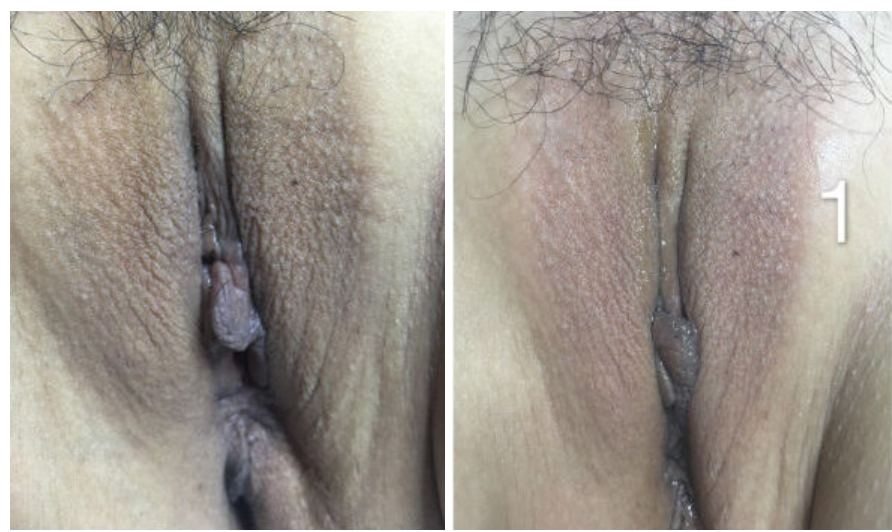

Figure 1. Baseline situation (left): quite severe 3-year vulvar atrophy; at right (marked with " 1 "): evolution of atrophy after the first DQRF session. Woman's age: 41; operational power: $8-10 \%$.

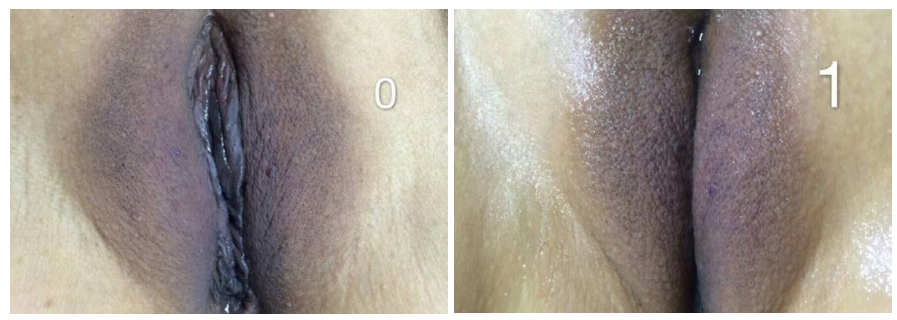

Figure 2: Baseline situation (at left, marked with "0"): 1-year yet rapidly evolving vulvar atrophy; at right (marked with "1"): evolution of atrophy after the first DQRF session. Woman's age: 35 ; operational power: $8-11 \%$.

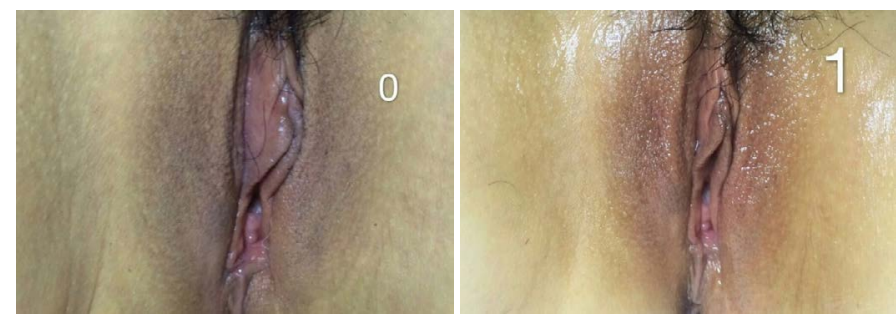

Figure 3. Baseline situation (left, marked with " 0 "): moderate yet steadily worsening 2-year vulvar atrophy; at right (marked with " 1 "), evolution of atrophy after the first DQRF session. Woman's age: 29 ; operational power: $10 \%$.
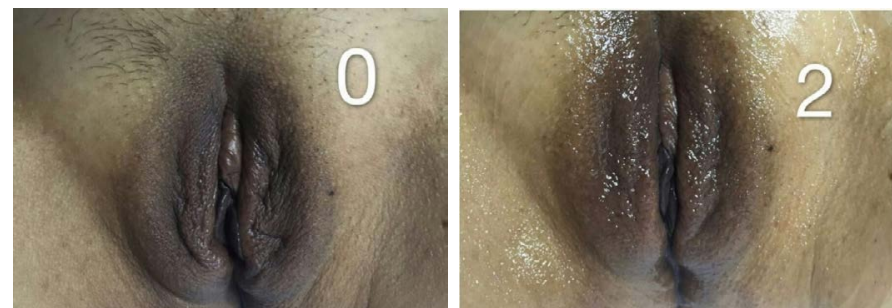

Figure 4. Baseline situation (left, marked with " 0 "): moderate vulvar 2.5-year atrophy; a right (marked with " 2 "), evolution of atrophy after the second DQRF session. Woman's age: 36 ; operational power: $9-12 \%$.
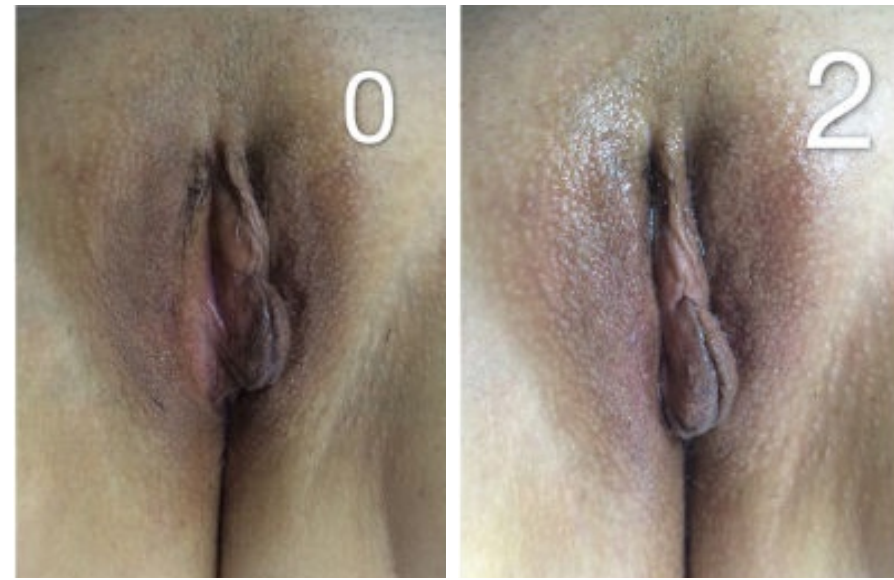

Figure 5. Baseline situation (left, marked with " 0 "): recent yet quite severe vulvar atrophy; at right (marked with "2"): evolution of atrophy after the second DQRF session. Woman's age: 31 ; operational power: $10-13 \%$.
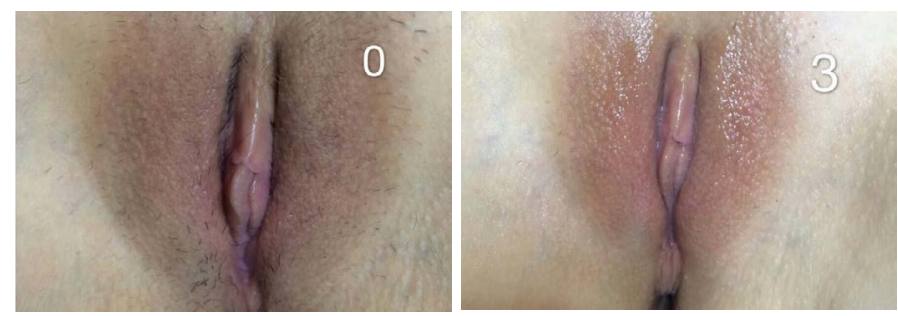

Figure 6. Baseline situation (left, marked with "0"): quite severe 1-year vulvar atrophy; right (marked with " 3 "), evolution of atrophy after the third DQRF session. Woman's age: 37 ; operational power: $8-12 \%$.

Table 1 (subjective appreciation of current vulvar aesthetics) and Table 2 (discomfort and self-esteem and couple-relationship problems incurred in daily life) illustrate the perceived levels of gratification reported by the sampled women before each of the four DQRF sessions and at follow-up interview. The distribution of categorical assessments showed a statistically significant shift compared with baseline towards more subjective satisfaction before the second rejuvenation session; the 

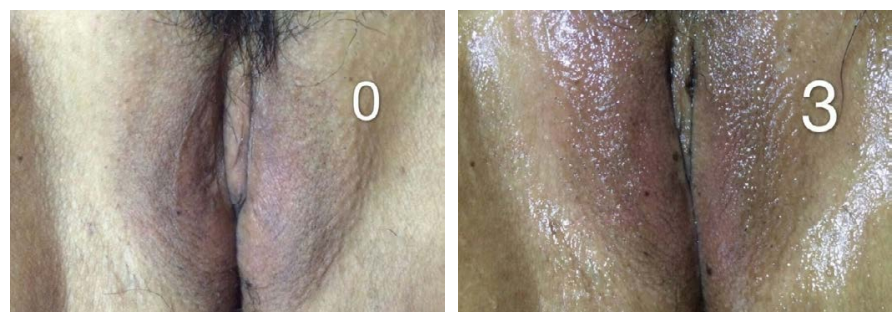

Figure 7. Baseline situation (left, marked with " 0 "): quite severe 3-years vulvar atrophy; a right (marked with "3"), evolution of atrophy after the third DQRF session. Woman's age: 40; operational power: $8-12 \%$.
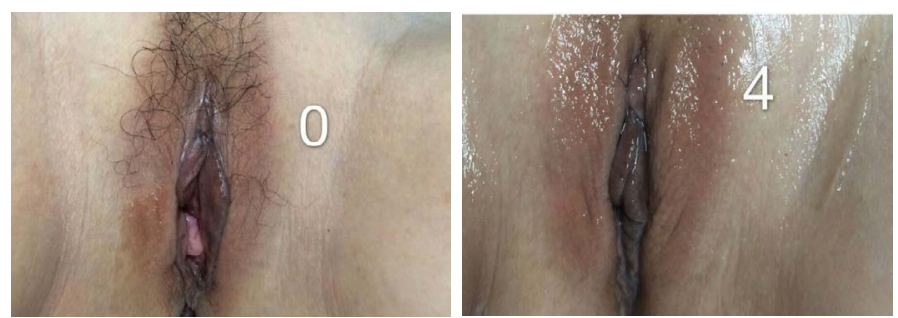

Figure 8. Baseline situation (left, marked with "0"): moderate to severe 2-year vulvar atrophy; at right (marked with "4"), evolution of atrophy 2 weeks after the fourth DQRF session. Woman's age: 34 ; operational power: $8-13 \%$

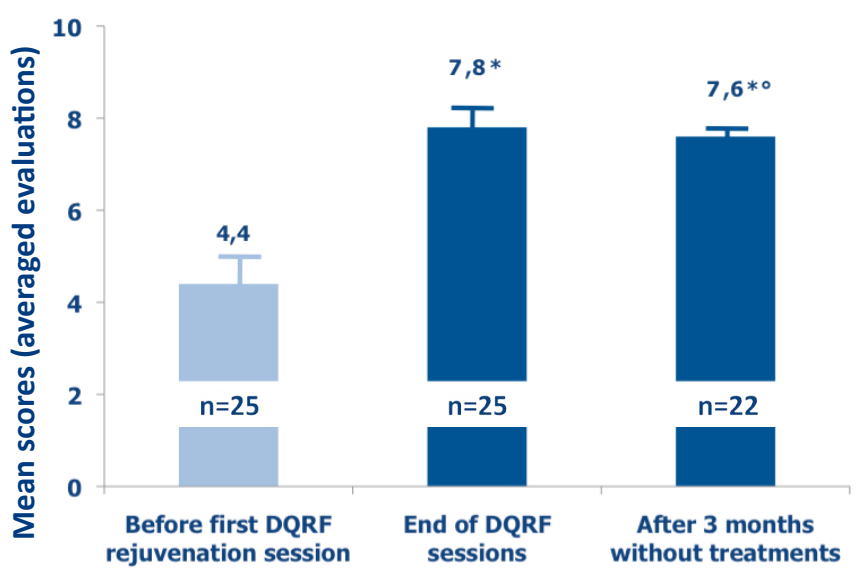

Figure 9. Mean averaged VAS scores ( \pm SEM) attributed to the aesthetic vulvar appearance before the first DQRF rejuvenation session (baseline), before the fourth and last DQRF session and 3 months after the end of the treatment cycle $\left({ }^{*} p<0,05\right.$ vs. baseline situation; ${ }^{\circ}$ no statistically significant difference vs. end of treatments).

Table 1. Distribution of women's VAS scores (subjectively perceived vulvar aesthetics) over the DQRF vulvar rejuvenation treatment cycle up to the last session $(n=25)$ and after 3 months without further treatments $(n=22)$.

\begin{tabular}{|c|c|c|c|c|}
\hline & $\begin{array}{c}\text { Not at all } \\
\text { satisfied }\end{array}$ & Poorly satisfied & Fairly satisfied & Highly satisfied \\
\hline $\begin{array}{c}\text { Before first } \\
\text { session }\end{array}$ & 16 & 9 & 0 & 0 \\
\hline $\begin{array}{c}\text { Before second } \\
\text { session* }\end{array}$ & 5 & 9 & 8 & 3 \\
\hline $\begin{array}{c}\text { Before third } \\
\text { session }\end{array}$ & 0 & 2 & 8 & 15 \\
\hline $\begin{array}{c}\text { Before last } \\
\text { session** }\end{array}$ & 0 & 1 & 8 & 16 \\
\hline $\begin{array}{c}\text { Follow-up } \\
\text { (3 months)** }\end{array}$ & 0 & 0 & 7 & 15 \\
\hline
\end{tabular}

$* \mathrm{p}<0.05, * * \mathrm{p}<0.01$, Chi-square test.
Table 2. Distribution of women's VAS scores (discomfort in everyday life, loss of self-esteem, problems with sexual life and couple relationship and other difficulties) over the DQRF vulvar rejuvenation treatment cycle up to the last session $(\mathrm{n}=25)$ and after 3 months without further treatments $(\mathrm{n}=22)$. Overall distribution, ${ }^{*} \mathrm{p}<0.05 ; * * \mathrm{p}<0.01$ vs. baseline (Chi-square test).

\begin{tabular}{|c|c|c|c|c|}
\hline & $\begin{array}{c}\text { Not at all } \\
\text { satisfied }\end{array}$ & Poorly satisfied & Fairly satisfied & Highly satisfied \\
\hline $\begin{array}{c}\text { Before first } \\
\text { session }\end{array}$ & 14 & 11 & 0 & 0 \\
\hline $\begin{array}{c}\text { Before second } \\
\text { session* }\end{array}$ & 4 & 9 & 11 & 1 \\
\hline $\begin{array}{c}\text { Before third } \\
\text { session }\end{array}$ & 0 & 1 & 15 & 9 \\
\hline $\begin{array}{c}\text { Before last } \\
\text { session }\end{array}$ & 0 & 0 & 8 & 17 \\
\hline $\begin{array}{c}\text { Follow-up (3 } \\
\text { months) }\end{array}$ & 0 & 1 & 5 & 16 \\
\hline
\end{tabular}

women's perceived satisfaction reached high statistical significance vs. baseline at the end of the DQRF cycle without appreciable deterioration over the following no-treatment months.

No treated woman reported any clinically significant or disturbing side effect or discomfort during the procedures. The operator reported, in almost all women, only a slight degree of hyperaemia and a subjectively pleasant, or at least undisturbing, warm sensation that largely resolved within 30 minutes and completely in a few hours.

\section{Discussion}

The physical effect of exposure to radiofrequency fields is induction of oscillating electrical currents in target tissues with translational motion of charged atoms and molecules and re-orientation of permanent dipole moments of water molecules. Viscosity of water translates into resistance (impedance) to molecular movements and rotations, leading to dissipation of motion energy and heat generation in female tissues [12].

Contraction due to breakage of intra-molecular hydrogen bonds and partial denaturation of collagen by radiofrequency fields is first seen at a tissue temperature of about $60^{\circ} \mathrm{C}$; collagen denaturation at about $67^{\circ} \mathrm{C}$ correlates with maximal signal to fibroblasts for neocollagenesis and it is frequently sought in dermatological medical procedures. Lower levels of tissue temperature $\left(40-45^{\circ} \mathrm{C}\right)$ are instead ideal for tightening and rejuvenation effects in skin and vulvar areas thanks to the long thermal relaxation time (about $225 \mathrm{msec}$ ) of collagen and other subepithelial vulvar structures $[12,15]$. A target temperature of $42^{\circ} \mathrm{C}$ in vulvar tissues as induced by the "EVA ${ }^{\mathrm{m}} \mathrm{Vulvar}$ Rejuvenation" protocol avoids triggering the pain threshold of vulvar nociceptors. Compared with laser technologies, deposition of new elastin is relatively unique to radiofrequency devices and gives peculiar mechanical strength and tightness, but also elasticity, to vulvar tissues $[12,15,16]$.

The 1.0-1.3 MHz DQRF generator is equipped with four stainless steel dynamic electrodes on anatomical probes (maximum emitting power, $55 \mathrm{~W}$ ). These quadripolar electrodes are continuously and electronically cycled between receiver and transmitter states. This hightech trick allows repelling electric fields to form that, when in the ideal combination, convey energy with high tridimensional precision to the subepithelial layers of the vulva.

This allows the operator to fine-tune the vulvar thermal effect in terms both of tissue volumes and depth, with the further benefits that the grounding pad on the upper thigh and the need for heavy 
energy burdens because of Ohm's resistances in tissues are eliminated. Low-energy vulvar rejuvenation is often pleasant with no downtime period and the risk of burns is virtually eliminated as shown in clinical studies in women with vaginal laxity and genitourinary syndrome of menopause carried out so far $[13,14]$.

As regards Aesthetic and Functional Gynaecology, a relatively new discipline for gynaecologists in spite of some dissenting opinion that is being occasionally heard $[17,18]$ and more and more practiced by plastic surgeons and specialists of aesthetic medicine 9 , the present study demonstrates the efficacy of new DQRF technology also when applied to vulvar rejuvenation. The photographic documentation visually shows that a tightening effect, even in women with quite severe vulvar atrophy, is clearly apparent already after the first or second treatment session. Objective VAS scores blindly attributed by authors almost doubled between the beginning and the end of the vulvar rejuvenation treatment sessions (from $4.2 \pm 0.45$ to $7.8 \pm 0.31, \mathrm{p}<0.05$ vs. baseline), strongly supporting the qualitative observation. Lack of a control group is a limit of the study design, yet dramatic aesthetic improvements look quite real.

The benefits experienced at the end of the DQRF rejuvenation cycle showed no appreciable tendency to dissipate over the following 3 months without further treatments, neither objectively nor in the subjective judgement of treated women. Noticeably, women's gratification for improved vulvar aesthetics, perceived psychological benefits, and reduced daily-life discomfort improved rapidly in the two weeks between the first and the second DQRF session and in the following two weeks before the third session. Subjective satisfaction of women steadily progressed until the end of the DQRF sessions and even in the following no-treatment period. The percent of women reporting to be fairly or highly satisfied increased from $92 \%$ after the third DQRF session to $96 \%$ after the fourth and last session and up to $100 \%$ after 3 months without further treatments ("Highly satisfied" women were $60 \%, 64 \%$ and $68 \%$, respectively). The trend was similar for self-perception and self-esteem, psychological consequences and impact on daily life and activities. Once again, lack of a control group may be another weak point of the study, but it does not invalidate its objective and subjective favourable outcomes.

Our results encourage us to look more in depth into the potential of a new technology that is easy to master and to practice in any private office, is free of any serious or disturbing complications and, as demonstrated retrospectively in this study, seems to reward treated women's expectations both in terms of subjective aesthetic gratification and self-esteem and impact on daily life. More studies are warranted about the cytological, histological and overall biological effects of the DQRF technology; expanding the number of women exposed to new technology should also be a goal. A similar goal should be providing more data related to very long-term follow-up periods: so far, safety and efficacy outcomes from clinical studies are available for one year $[13,14]$. Validated questionnaires will have to be used to assess the subjective level of gratification of treated women.

\section{Conflict of Interest Disclosures}

Gianluca Benincà, David Bosoni and Franco Vicariotto are Medical Consultants and members of the Scientific Board of Novavision Group S.p.A. (Misinto, Monza-Brianza, Italy), manufacturer of the DQRF technology used during the investigation. Mauro Raichi is a Medical Research Consultant for Novavision Group S.p.A. (Misinto, MonzaBrianza, Italy).

\section{References}

1. Committee Opinion No. 662 (2016) Breast and Labial Surgery in Adolescents. American College of Obstetricians and Gynecologists. Obstet Gynecol 127: e138-140.

2. Portman D, Gass M, Vulvovaginal Atrophy Terminology Consensus Conference Panel (2014) Genitourinary syndrome of menopause: new terminology for vulvovaginal atrophy from the International Society for the Study of Women's Sexual Health and The North American Menopause Society. Menopause 21: 1063-1068. [Crossref]

3. Krychman ML (2016) Vaginal Laxity Issues, Answers and Implications for Female Sexual Function. J Sex Med 13: 1445-1447. [Crossref]

4. Goodman MP, Placik OJ, Benson RH $3^{\text {rd }}$, Miklos JR, Moore RD, et al. (2010) A large multicenter outcome study of female genital plastic surgery. J Sex Med 7(4 Pt 1): 15651577. [Crossref]

5. Liao LM, Creighton SM (2007) Requests for cosmetic genitoplasty: how should healthcare providers respond? BMJ 334: 1090-1092. [Crossref]

6. Italian Association for Plastic Aesthetic Surgery (AICPE), Italian Association for Aesthetic and Functional Gynaecology (AIGEF). Joint Session, 2nd National AICPE Congress, 14-16 March 2014, Florence (Italy)

7. Foldes P, Droupy S, Cuzin B (2013) [Cosmetic surgery of the female genitalia]. Prog Urol 23: 601-611. [Crossref]

8. Triana L, Robledo AM (2015) Aesthetic surgery of female external genitalia. Aesthet Surg J 35: 165-177. [Crossref]

9. Vanaman M, Bolton J, Placik O, Fabi SG (2016) Emerging Trends in Nonsurgical Female Genital Rejuvenation. Dermatol Surg 42: 1019-1029. [Crossref]

10. Sekiguchi Y, Utsugisawa Y, Azekosi Y, Kinjo M, Song M, et al. (2013) Laxity of the vaginal introitus after childbirth: nonsurgical outpatient procedure for vaginal tissue restoration and improved sexual satisfaction using low-energy radiofrequency thermal therapy. $J$ Womens Health (Larchmt) 22: 775-781. [Crossref]

11. Gambacciani M, Levancini M, Cervigni M (2015) Vaginal erbium laser: the secondgeneration thermotherapy for the genitourinary syndrome of menopause. Climacteric 18: 757-763. [Crossref]

12. Tadir Y, Gaspar A, Lev-Sagie A, Alexiades M, Alinsod R, et al. (2017) Light and energy based therapeutics for genitourinary syndrome of menopause: Consensus and controversies. Lasers Surg Med 49: 137-159. [Crossref]

13. Vicariotto F, Raichi M (2016) Technological evolution in the radiofrequency treatmen of vaginal laxity and menopausal vulvovaginal atrophy and other genitourinary symptoms: first experiences with a novel dynamic quadripolar device. Minerva Ginecol 68: 225-236. [Crossref]

14. Vicariotto F, De Seta F, Faoro V, Raichi M (2017) Dynamic quadripolar radiofrequency treatment of vaginal laxity/menopausal vulvo-vaginal atrophy: 12-month efficacy and safety. Minerva Ginecol 69: 342-349. [Crossref]

15. Beasley KL, Weiss RA (2014) Radiofrequency in cosmetic dermatology. Dermatol Clin 32: 79-90. [Crossref]

16. Alexiades M, Berube D (2015) Randomized, blinded, 3-arm clinical trial assessing optimal temperature and duration for treatment with minimally invasive fractional radiofrequency. Dermatol Surg 41: 623-632. [Crossref]

17. Hailparn TR (2012) Cosmetic gynecology and the elusive quest for the "perfect" vagina. Obstet Gynecol 120: 1207-1208. [Crossref]

18. Cain JM, Iglesia CB, Dickens B, Montgomery O (2013) Body enhancement through female genital cosmetic surgery creates ethical and rights dilemmas. Int J Gynaecol Obstet 122: 169-172. [Crossref]

Copyright: (C2017 Benincà G. This is an open-access article distributed under the terms of the Creative Commons Attribution License, which permits unrestricted use, distribution, and reproduction in any medium, provided the original author and source are credited. 